\title{
Nanometric Surface Oscillation Spectroscopy of
}

\section{Water-Poor Microemulsions}

Mario Corti ${ }^{1}$, Antonio Raudino ${ }^{2}$, Laura Cantu ${ }^{3}$, Johannes Theisen $^{4} *$, Maximilian Pleines ${ }^{5}$, Thomas Zemb ${ }^{5}$

\author{
${ }^{1}$ CNR-IPCF, Viale Ferdinando Stagno d'Alcontres 37, 98158 Messina, Italy \\ ${ }^{2}$ Dept. Chemical Science, University of Catania, Viale A. Doria 6, 95125 Catania, Italy \\ ${ }^{3}$ Dept. Medical Biotechnology and Translational Medicine, University of Milano, LITA, \\ Via Fratelli Cervi 93, 20090 Segrate, Italy \\ ${ }^{4}$ ICSM CEA/CNRS/UMontpellier/ENSCM, CEA Grenoble, 17 Avenue des Martyrs, 38000 \\ Grenoble, France \\ ${ }^{5}$ ICSM CEA/CNRS/UMontpellier/ENSCM, CEA Marcoule, BP17171, 30207 Bagnols-sur-
} Cèze, France

\author{
KEYWORDS \\ Solvent extraction, Droplet surface waves, Fabry-Perot Interferometry, Ion recognition, \\ Extractant phase behavior
}

ABSTRACT 
Selectively exchanging metal complexes between emulsified water-poor microemulsions and concentrated solutions of mixed electrolytes is the core technology for strategic metal recycling. Nanostructuration triggered by solutes present in the organic phase is understood, but little is known about fluctuations of the microemulsion-water interface. We use here a modified version of an opto-electric device initially designed for air bubbles, in order to evidence resonant electrically induced surface waves of an oily droplet suspended in an aqueous phase. Resonant waves of nanometer amplitude of a millimeter-sized microemulsion droplet containing a common ion-specific extractant diluted by dodecane and suspended in a solution of rare earth nitrate are evidenced for the first time with low excitation fields $(5 \mathrm{~V} / \mathrm{cm})$. From variation of the surface wave spectrum with rare earth concentration, we evidence up-take of rare-earth ions at the interface and at higher concentration the formation of a thin "crust" of liquid crystal forming at unusually low concentration, indicative of a surface induced phase transition. The effect of the liquid crystal structure on the resonance spectrum is backed up by a model, which is used to estimate crust thickness. 
Refining or recycling rare and precious metals in the form of concentrated electrolyte solutions, an important issue in modern technology, can be achieved by liquid-liquid extraction and stripping ${ }^{1}$. Emulsified droplets, with size $10^{-3}$ to $10^{-2} \mathrm{~m}$, are formed in mixer-settlers or in pulsed columns in order to favor selective transfer back and forth of the "target" metal through the wide dispersed interface of close contact between the water-poor microemulsion droplets and the feed aqueous phase where the "waste" is left. Selectivity between "target" and "waste" electrolyte must be as high as possible, but extraction and desextraction reasonably driven by concentration, $\mathrm{pH}$, salinity, oxidation state of the present species.

Hence, the practical problem: Up to now, existing technologies leading to an efficient phase transfer are not sufficiently developed to provide cost-effective recycling taking into account the principles of green chemistry. In the case of rare earths to be separated from iron, as in the practical case of recycling neodymium magnets, no economical and chemically acceptable process is currently available. Therefore, less than $1 \%$ of the corroded magnets are currently recycled. This is largely due to a lack of understanding of the thermodynamics and the kinetics of ion transfer between an aqueous solution in equilibrium with a water-poor microemulsion containing an oil-soluble weak surfactant, named "extactant" in formulators" language.

While thermodynamic equilibrium between salts in the two coexisting phases is relatively well understood, very little is known concerning the liquid-liquid interface: ion speciation, presence of mono- or multilayers of surface active agents and multi-cationic complexes at the interface, for instance. Indeed, the ion transfer occurs via an "interphase" that can be either a single molecular layer, or a multiple layer ${ }^{2,3}$ or even a thicker interphase described as "hazing" course, emulsification of millimeter-sized droplets is used to maximize the contact for exchange of species improving the efficient transfer of metallic cations: from water to oil in the 
"extraction" section and the reverse in the "stripping" section. Still, exhaustive testing at pilot scale is currently required, for optimal working conditions to be selected. To understand the molecular and colloidal mechanisms occurring at the exchanging interphase, a minimal model considers the liquid-liquid interface as composed of the surface-active complexant molecules present in the oil-rich phase $\mathrm{e}^{5}$ but also the "Stern" complexed cation layer6 and the diffuse layer of counter-ions extending typically over Debye and Guy-Chapman lengths, in the nanometer range ${ }^{7}$.

On the other hand, there is a lack of methods characterizing the dynamics of interfacial layers in the length scale from 1 to $100 \mathrm{~nm}$. To characterize this thick liquid-liquid interface that can vary from a molecular monolayer to a macroscopic crust with liquid crystal structure upon approaching the phase boundary, we have modified an experimental setup initially designed for detecting oscillations of an air bubble immobilized in an aqueous solution ${ }^{8}$. We use a confocal Fabry-Perot optical arrangement $(\mathrm{CFP})^{9}$ working near the equatorial plane of a mm-sized droplet immersed in an immiscible fluid, which allows direct access to amplitude, frequency and quality factor of electrically induced oscillations of the droplet. The droplet is initially a water-poor microemulsion, so-called "Winsor II" state, made of dodecane in which a typical concentration of extractant used in hydrometallurgical processes is dissolved, and a few water molecules. It is immersed in a water-bath containing the rare earth to be extracted. According to general behavior about surfactants at interfaces, the interphase at the macroscopic interface contains at least one monolayer ${ }^{10}$, but this was never quantified directly, to our knowledge. At that extractant concentration, electrolytes are adsorbed at the droplet via specific recognition until equilibrium is attained with the "excess" aqueous solution at fixed $\mathrm{pH}$ where the lanthanide is dissolved as nitrate salt ${ }^{11}$. We use the very common extractant tetraoctyldiglycolamide 
(TODGA) used for rare earth and actinide separation ${ }^{12}$, owing to the high affinity of the diglycolamide "head"13,14. Like all other neutral extractants used in practice, TODGA is an oilsoluble surface-active agent and therefore also a "weak" surfactant: the critical micelle concentration $(\mathrm{CMC})$ in the oil phase is beyond $0.1 \mathrm{M}^{15}$. In the series of experiments here presented, the Europium nitrate concentration of the water-bath is progressively increased. In all known ternary systems containing electrolytes at high aqueous concentration, a diluent and extractant at high concentration, the equilibrium bulk oily phase contains concentrated liquid crystalline phases (so-called "third phases") producing either broad or thin quasi-Bragg peaks, depending on the correlation between layers in a gel state. The model extractant used in this work follows the general rule. Tentative crystallization of metal complexed by long chains often results in the production of amorphous viscous material similar in content and X-pattern to third phase. Most of the time, a viscous "third" phase is obtained upon systematic testing of solvent composition and temperature ${ }^{16}$.

We show hereby that the measurement of amplitude, frequency and quality factor of the resonant surface waves versus Europium nitrate concentration in the reservoir, allows a) to directly determine the adsorption of Eu mainly in the form of uncharged quadruplet complex at the droplet surface and b) to detect the formation of a micrometer-thick "crust" of liquid crystalline material obtained when the Europium concentration approaches bulk phase boundary concentration.

\section{RESULTS AND DISCUSSION}


The experimental device used to investigate surface effects induced in the event of rare earth recognition by an uncharged molecule (i.e. complexation of Europium with TODGA electronegative head-group ${ }^{17}$ ) is a modified version of the device recently described by Cantu' and coworkers. This confocal Fabry-Perot device is able to detect, without calibration, capillary waves with amplitudes of the order of one nanometer excited on the surface of a mm-sized drop or bubble, around the natural frequency, $\sim 100 \mathrm{Hertz}^{18}$. The electro-acoustic excitation of these oscillations is made via a weak electric field of the order of $5 \mathrm{~V} / \mathrm{cm}^{19}$ and is imposed by two electrodes located below the bubble or drop and on the hollow tube above ${ }^{20}$.

The modified instrument used in this work examines mm-sized droplets of an insoluble "oil" phase, namely a water-poor extractant microemulsion, immersed in an aqueous solution containing electrolytes, namely Europium nitrate. If excited, resonant oscillations with amplitudes of the order of one nanometer are negligible versus the diameter of the droplet, so that the interface is flat at molecular length-scale, and offers one complexation site per $\mathrm{nm}^{2}$ of interface.

The main features of this instrument are presented in Figure 1. Surface waves, if present, are detected as fluctuations of the diameter of the droplet, that is, of the optical path difference between the two reflections of a traversing laser beam originated at the income and outcome spots on the drop surface. This confocal Fabry-Perot configuration, set near the droplet equator ${ }^{9}$, ensures high sensitivity. Full dark-to-bright passage on the interference fringe pattern corresponds to $\lambda / 8$ variation in the radius of the drop, $79 \mathrm{~nm}$ in our case, and variations of the order of $1 \mathrm{~nm}$ can be detected. This configuration was shown to develop a better sensitivity to 
very small surface adsorptions ${ }^{21}$, and to be more sensitive than interferometry using interfacial refraction used to investigate living cell membrane fluctuations ${ }^{22}$.

The details of the experimental detection of resonance frequencies, amplitudes and quality factors are found in the initial description of the instrument ${ }^{18}$. The electrical excitation frequency is swept slowly (ca. one minute) in the range from 50 to $500 \mathrm{~Hz}$. Raw data output consists in an amplitude-to-frequency spectrum showing peaks in correspondence to the resonance frequencies of the extractant microemulsion droplet, for different Europium nitrate concentrations in the aqueous phase. The quality factor, given by the FWHM of the resonance, is nearly constant in the investigated range of concentrations, and close to 2 . We notice that in the case of trivalent lanthanides cations, even a solution with molarity $C_{\mathrm{M} / 1}=10 \mathrm{mM}$ is considered as concentrated. In the case of Europium nitrate, by taking into account an osmotic coefficient, $\varphi$, close to 0.8 and the number of particles produced by dissociation of an Europium nitrate salt, $n=4$, the osmolarity is calculated as $C_{\mathrm{osm}}=\varphi n C_{\mathrm{M} / 1}=\gamma \cdot C_{\mathrm{M} / 1}=30 \mathrm{mosm} / \mathrm{L}$, with $\gamma$ close to $3 .{ }^{23}$

The solvent droplet examined in this work has a typical composition used in industrial liquidliquid extraction processes, such as rare earth extraction for recycling magnets from windmills ${ }^{24}$. In industrial plants using cascade-coupled mixer-settler tanks or pulsed extracting columns, the "solvent phase" consists of an oil-soluble weakly surface-active extractant and a waterimmiscible fluid called "diluent"; this "oil" phase is contacted with a concentrated solution of mixed ions in the presence of an acid with the same counter-ion ${ }^{11}$. In any efficient hydrometallurgical process, acids are always present in large quantities, since they are used in steps previous to liquid-liquid extraction, i.e. during leaching or dissolution. Moreover, using 
high quantities of nitric acid has two advantages. First, it triggers formation of water-poor microemulsions, i.e. small reverse water-in-oil micellar aggregates ${ }^{25}$. The mechanism is that of reducing the amount of "inactive" monomers of complexants, i.e. not involved in the reverse aggregates, thus favoring the solvent phase during extraction ${ }^{26}$. This tendency of triggering aggregate-formation is a general property of chaotropic anions, as present in the Hofmeister series, such as nitrates, as well as "antagonistic" salts made by a chaotropic-cosmotropic couple $^{25}$. Second, when extractants are used in synergetic conditions, there is a pronounced "peak" in the target/remaining cation selectivity occurring at low $\mathrm{pH}$, allowing optimization of the yield of efficient process implementation ${ }^{27}$.

As an example, a target electrolyte (in our case Europium nitrate) water/solvent equilibrium partition coefficient of ten $(5 \mathrm{~kJ} /$ mole free energy of transfer) towards the solvent phase, together with negligible concomitant transfer of iron (less than $0.1 \%$ ), is necessary to obtain a robust process, that can be used for recycling of rare earths separated from iron, the two being admixed in concentrated acids after dissolution of the oxide from permanent magnets. In optimized conditions, a "moderate" chemical equilibrium, with a final distribution coefficient close to ten in favor of the solvent phase, is necessary. In fact, for larger values of distribution coefficients, the efficiency of the whole process would decrease, since stripping would be hindered in the subsequent desextraction stage.

Up to now, in practical cases, only trial and error essays, varying temperature and concentration of extractant, allow to design a process chart, implementing the "best" conditions maximizing the yield of extraction and desextraction in a "cascade" arrangement, while keeping the rare earth/iron selectivity high. Since nitrate molecules are counter-ions of lanthanides to be extracted, high nitric acid concentration, well above one molar, is used to optimize yields ${ }^{11}$. 
The droplet of solvent phase as seen in Figure 1 (A and B) is the water-poor microemulsion ${ }^{28}$ in equilibrium with the excess aqueous solution of fixed $\mathrm{pH}$ where the Europium concentration is progressively increased. The solvent droplet can exchange Europium nitrate with nitric acid at $\mathrm{pH}=3.3$. A variation of the drop resonance frequency reveals adsorption of Europium at the interface between the water-poor microemulsion droplet and the bulk aqueous phase, due to "recognition" of the rare earth by the diglycolamide heads of the extractant. Equilibrium is reached typically within 10 to 20 minutes after changing the electrolyte concentration in the reservoir. Once Europium cations are in equilibrium in the two phases present, no swelling or deswelling of the solvent phase droplet occurs. If present, this volume variation would be observed as a slow outwards or inwards shift of the fringe pattern.

The content of Europium nitrate in the aqueous phase filling the sample cell (Fig 1C) is close to the "feed" treated in industrial processes, differing only by the absence of an overwhelming concentration of ferric nitrate, present in industrial separation plants. Industrial-scale extraction is typically worked at lower $\mathrm{pH}$ than in the experiment described in this work, that is, close to $\mathrm{pH}$ $=1$ instead of $\mathrm{pH}=3.3$. With the electro-acoustic excitation as described in this experiment, $\mathrm{pH}$ values lower than 3 are not accessible because of Joule heating of the reservoir. So, an intermediate $\mathrm{pH}=3.3$ was chosen as a best compromise between conditions used in industrial practice and applicability of the electrical excitation of surface waves in the CFP drop interferometer.

Figure 2a shows a typical surface wave resonance peak obtained for one given composition of the aqueous phase in the reservoir, namely $0.79 \mathrm{mM}$ Europium solution at $\mathrm{pH}=3.3$. In the 
frequency domain close to the surface wave resonance, an increase in the inverse of quality factor reflects the efficiency of the dissipation mechanism. Since the shape of the resonance peak is well fitted by a Lorentzian, there is only one dominant relaxation mechanism occurring, and the broadening is not due to inhomogeneities.

In Figure $2 \mathrm{~b}$ we present the raw amplitude-versus-frequency spectra obtained for a series of experiments performed on a same drop of solvent in solution of different Europium nitrate concentrations at fixed $\mathrm{pH}=3.3$, adjusted with nitric acid. The concentration of Europium in the reservoir is increased by small steps in the range from 0 to $45 \mathrm{mM}$. When lanthanide concentration in the reservoir is close to $45 \mathrm{mM}$, the droplet becomes turbid and iridescent, due to the presence of an evident liquid crystal structure. At this point, the turbidity of the droplet constitutes a limitation to the optical interferometer, still the drop interface oscillates and a measurable frequency is detected up to this limit.

Operating in a test tube, i.e. on bulk volumes, upon increasing the lanthanide concentration in the aqueous phase to this limit $(45 \mathrm{mM}$ at $\mathrm{pH}=3.3)$, the sample becomes a stiff bi-refringent gel, corresponding to a three-phase multiple emulsion that shows separation only after extensive centrifugation. Below that maximum admissible lanthanide concentration, a clear oily phase is located above the excess water phase. This is a Winsor II to Winsor III regime transition known as the third phase transition in chemical engineering. In a water-poor microemulsion the solvent is in equilibrium with an excess solution containing electrolytes. This practical distinction between surfactants (extractants) and hydrotropes was introduced by Schulman ${ }^{29}$. Four classes of microemulsions differ from the "hydrotropy" concept as proposed by Winsor ${ }^{30}$. The conditions chosen in hydrometallurgic processes are always water-poor microemulsions in 
the Winsor II regime and use only hydrotropes as "solvent modifiers"31. Conversely, the Winsor III regime, where the sample spontaneously splits into three phases instead of two after a liquidliquid separation of the solvent phase, must be avoided at any cost. In fact, the change of viscosity linked to spontaneous emulsification occurring upon the Winsor II-Winsor III transition would stop any industrial plant from operation. This transition is triggered by effective attraction between polar cores of the reverse aggregates, exchange of ions between cores as well as by coalescence of reverse aggregates ${ }^{32}$.

The Winsor II to Winsor III transition occurs at slightly lower concentrations in pulsed columns for rare earth extraction than in the "bulk" of a test tube with only few $\mathrm{ml}$ of each phase present. A main point is that in plants the solvent phase is not "bulk" but dispersed as small emulsion droplets suspended in water, with mm-to-cm typical size, with much higher surface-tovolume ratio. This suggests that this condensation transition from concentrated extractantextracted complexes towards a liquid crystalline phase is favored by the locally flat $\mathrm{O} / \mathrm{W}$ interface constituting a template for the new formed phase, in a surface-induced phase transition. The plant situation is similar to the configuration of the mm-drop in the CFP interferometer.

After the test tube assay, the condensed "third phase" produced upon upper-limit Europium complexation could be separated from excess water and solvent and it could be analyzed separately by X-ray scattering (SWAXS), viscosimetry and Young modulus (see Suppl. Inf.). Nanostructural analysis by SWAXS, shows that the "third phase" consists of a liquid crystal with smectic order, with a periodicity of $2.16 \mathrm{~nm}$, close to the TODGA molecular length. In fact, the SWAXS spectrum shown in Figure 3 exhibits three sharp peaks (1, 2, 3 lamellar repetitions) and 
a WAXS profile typical for molten hydrocarbon chains. The layered structure is well developed and the local order of alkyl chains in this phase is very similar to that in pure dodecane at the same temperature $\left(T=25^{\circ} \mathrm{C}\right)$, slightly more compact. From the observed $q$-position of the broad bump and of the sharp Bragg peaks, in the SAXS region $q<10 \mathrm{~nm}^{-1}$, and of the broad liquid band associated to molten chains, in the WAXS region $q>10 \mathrm{~nm}^{-1}$, a $2: 1$ stoichiometric TODGA:Eu ratio can be inferred, with a very low dodecane content, of the order of a few percent.

Co-extracted water, in most cases studied of the order of one water molecule per extractant and one to four water molecules per extracted cosmotropic ion, is typical for most third phases for which it was derived from limits of stability in the phase diagram ${ }^{33}$. Water molecules fractionated in "bound" first layer of the extractant and "free" water inside the liquid crystal designed as third phase is usually less than $10 \%$ of the total. The nearby located "water-poor microemulsion" has only a low amount of water. The head-group area per extractant in the smectic liquid crystalline phase is expected to be similar to the one at the microemulsion phase boundary. In the latter case, it is known from w/o surface tension experiments to be close to 1 $\mathrm{nm}^{2}$ (See Suppl. Inf. Section 2).

Back to the drop experiment, the important features of the resonance spectra shown in Figure $2 \mathrm{~b}$ are summarized in Figure 4: three different regimes can be identified. First, the resonance frequency decreases upon lanthanide addition until a second regime occurs, where it keeps a constant value. Finally, for lanthanide bulk concentration above $25 \mathrm{mM}$, the resonance peak shifts again towards higher frequency. As already pointed out, above $45 \mathrm{mM}$ lanthanide in the 
bulk water phase, the droplet becomes opaque to the incident light, as shown by the photography in Fig. 1F.

In the first regime $\left(C_{\mathrm{Eu}}\right.$ up to $\left.10 \mathrm{mM}\right)$, the downshift of the resonance frequency reveals a decrease in surface tension of the droplet. This behavior is expected, in the case that electrolytes are taken up by the complexant ${ }^{34}$. In fact, an increase of surface disjoining pressure, connected to adsorption of trivalent ions or other cationic nitrate complexes at the interface, is occurring. So, the observed frequency downshift allows estimating the charge of adsorbing ions. To our best knowledge, the speciation of the species adsorbed at the water/microemulsion droplet interface could not yet be determined experimentally by any available technique.

We notice that in the bulk, in this range of $\mathrm{pH}$ and concentration, Eu ions are known to be $90 \%$ as trivalent cations and $10 \%$ as divalent Europium monodentate, with a negligible amount of monovalent Europium dinitrate and neutral Europium trinitrate ${ }^{23}$. Hence, highly charged trivalent europium ions and some mono-nitrate complexes are usually thought to progressively adsorb to the surface, producing a significant disjoining pressure even for small adsorbed amounts, due to electrostatic lateral repulsion. Moreover, there is an interplay between complexation and electrostatic interaction. To calculate the speciation using surface tension values, the self-consistent "charge regulation model" introduced by Ninham and Parsegian was used, largely known for similar quantifications since 1971. This model considers interactions occurring not only between an ion and its recognizing site, but also with the ions already adsorbed on the "neighboring" sites. Then, a long range colloidal electrostatic term is added to the free energy, thus distinguishing chemisorbed and physico-sorbed ions at the interface ${ }^{35}$. This 
quantitative approach was used successfully in a large variety of colloidal systems ${ }^{36}$ : emulsions, microemulsions, micelles and liquid crystals, such as adsorption of halides on phospholipids ${ }^{37}$.

Figure 5a, shows the experimentally observed decrease in surface tension. Experimental values are measured at the lowest drop resonance, $l=2$, and calculated using resonance frequency values and the frequency-surface-tension relationship mentioned as equation (19) in supplementary information. The experimental measurement is shown together with the dependence on bulk $\mathrm{Eu}$ concentration expected in the case of bare trivalent cations and mononitrate complexes, assumed with the same speciation as known in the bulk $\left(\mathrm{Eu}^{3+}: \mathrm{Eu}^{2+}=\right.$ 9:1). It is obvious that the observed and the expected are totally incompatible. The chemically/physically balanced adsorption on the surface of such complexes, dominant in the "bulk", would not produce the large decrease in surface tension as observed. In the same figure, behaviors expected for trivalent, divalent, monovalent and neutral species are shown. Indeed, any adsorption of highly charged species at the interface can be excluded. Only uncharged species, possibly with a small amount of monovalent dinitrate complexes, with a free energy of complexation of $2.5 k_{\mathrm{B}} T /$ molecule $(7.5 \mathrm{~kJ} /$ mole) can produce the observed reduction of surface tension. Their effect can be modeled at first approximation via a Langmuir isotherm.

The Europium occupancy per extractant site on the drop surface is shown in Fig 5 b as a function of lanthanide concentration in the aqueous phase. The free energy of adsorption/desorption process of Europium, directly derived from the binding constant $\ln k=$ $\mathrm{DG}_{\mathrm{b}} / \mathrm{RT}$, associated to a Stern layer thickness with a typical complexation distance taken as diameter of the hydrated ion $(0.5 \mathrm{~nm})$, is found to be $2.5 k_{\mathrm{B}} T /$ molecule. This is a factor of ten lower than the complexation enthalpy derived from calorimetry ${ }^{38}$ and shows that 
enthalpy/entropy compensate in part when Europium is adsorbed at the interface ${ }^{39}$. As can be seen in Figure 5a, the order of magnitude of the measured surface tension is incompatible with the presence of any adsorbed charged species at the interface, either trivalent Europium ions, divalent $\mathrm{Eu}\left(\mathrm{NO}_{3}\right)^{++}$or even monovalent dinitrate complexes, that are known to be dominant in the bulk.

The dominant complex adsorbed at the interface must therefore be a neutral ion "quadruplet" as a neutral Europium trinitrate. The presence of some minor amount of monovalent Europium dinitrate complexes, driven by "matching affinity" mechanisms ${ }^{40}$ described in first approximation by a Langmuir isotherm, cannot be totally excluded, since it would only have a minor effect on surface tension, rather affecting the fluctuation amplitudes, that were nonetheless qualitatively comparable from one measurement to the other. Anyway, assuming identical "speciation" in the bulk and at the interface - a common assumption in current modeling of extraction yields in chemical engineering - is fundamentally inconsistent with the observed behavior.

At about $10 \mathrm{mM} \mathrm{Eu}$ concentration in the bulk, the process of surface filling has come to the final occupancy of $0.95 \mathrm{Eu}$ molecules $/ \mathrm{nm}^{2}$, almost the same surface per molecule of complexant, as shown in Figure 5b. From $10 \mathrm{mM}$ on, ion take-up adopts a steady state in the second regime shown on Figure 4.

We now turn to the third regime, starting at about $25 \mathrm{mM}$ concentration of Europium in the aqueous medium. The clear upward shift of the resonance frequency reveals an increase of the effective surface tension (or equivalently, a decrease of the apparent surface disjoining pressure). 
This effect is explained by the formation and growth of a thin crust made of the material that is the most stable state, a "third phase", i.e., a smectic liquid crystal that exists in the bulk in similar conditions, but at higher concentration of lanthanide.

Modeling of the shift in effective surface tension, $\gamma_{\text {eff }}$, can be done for different working hypotheses ${ }^{41}$ concerning the "crust" forming progressively upon increasing lanthanide concentration in the reservoir. It can behave as a lubricated sliding layered liquid crystal, as a viscous liquid or as a homogeneous isotropic elastic material of known Young modulus upon a low-viscosity core. The expressions of the resonance frequencies expected in these three cases are given in the Suppl. Inf. Materials Section 1. We find that the hypothesis of an elastic isotropic solid of varying thickness $d$ is compatible with the experiment. This finding, together with the observed smectic structure of the third phase revealed by SAXS, suggests that the forming crust is constituted by a physically cross-linked layered system, with a thickness that can be determined using experimental parameters.

The expression of resonance frequency induced by the presence of an isotropic crust forming at the drop interface only depends on the Young modulus and the thickness of the crust:

$f_{l m}=\frac{1}{2 \pi} \operatorname{Im} \omega_{l m}=\frac{1}{2 \pi} \sqrt{\frac{\gamma_{e f f}(l)}{R^{3}} \frac{(l+2)(l+1) l(l-1)}{\rho_{\mathrm{int}}(l+1)+\rho_{e x t} l}}$

where

$\gamma_{e f f}(l) \equiv \gamma_{O W}+\frac{\kappa_{M}^{e f f}}{R^{2}} l(l+1)$

The effective bending rigidity of the drop, $\kappa_{M}^{e f f}$, is an increasing function of the thickness $d$ and Young modulus of the crust, as reported by eq. (6) of Suppl. Inf. Section 1. 
Figure $5 \mathrm{c}$ shows the frequency shift of the lowest energy mode $l=2$ expected for different thicknesses of the crust, compared to experimental data. The experimental points are well distributed in this graph, and show that the surface crust inside the extractant/solvent drop nucleates at about $25 \mathrm{mM}$ of Europium in the reservoir. Upon progressive addition of Europium nitrate in the reservoir, the experimental points cross the curves expected for different crust thicknesses. Figure 5d shows a graphical determination of the thickness of the crust, plotted versus $\left(\mathrm{Eu} / \mathrm{Eu}^{*}\right)$, that is, the ratio between the actual concentration of Europium in the aqueous phase in contact with the drop and the concentration at which the onset of third phase is observed in the "bulk" test tube experiment, $\mathrm{Eu}^{*}=45 \mathrm{mM}$. The crust typically reaches a thickness of the order of $70 \mu \mathrm{m}$ when $\mathrm{Eu}$ in the bulk approaches the phase boundary.

A sketch of the phenomena occurring in the filling regime (Eu concentration $<10 \mathrm{mM}$ ) and in the crust formation regime (Eu concentration $>25 \mathrm{mM}$ ) is illustrated in Figure 6 together with the main parameters used in the modeling.

\section{CONCLUSIONS AND OUTLOOK}

To our knowledge, oscillations with amplitude lower than one micron of an oily droplet suspended in a reactive aqueous reservoir were not measurable before by any experimental method other than the confocal Fabry-Perot described in this work. The smallest oscillations described up to now in the literature on a pendant drop covered by an interphase were imaged in the micrometer range ${ }^{41,42 \cdot 43}$. The new technique introduced here increases experimental sensitivity by a factor of $10^{3}$ : even at the scale of typical distances coming in, all stress-strain 
interfacial relations can be assumed with safety as being linear. This high-resolution Fabry-Perot interferometry is exploited to study equilibrium thicknesses in the nanometer range of polymer brushes at solid-liquid interfaces ${ }^{44}$. Interferometry at the water interface was also used to detect patchy phase separation in the case of mucus coexisting with electrolytes ${ }^{45}$.

Here we addressed the resilience of operation of liquid-liquid extraction devices, involving the optimization, in terms of both yield and sustainability, in the formulation of fluids used in hydrometallurgy for the extraction and recycling of strategic metals. The experiment provided information of prominent importance.

First, it unraveled that speciation of lanthanides in the bulk and at the oil-water interface are qualitatively different: the trivalent Europium dominate in the bulk while the neutral trinitrate complex makes most of the droplet interface. Moreover, a decrease in kinetics occurs with approximately half of the maximum rare earth load that is determined by bulk phase diagram examination. This is especially important when complexants are used in synergy, since mixed extractant aggregates may select different complexes ${ }^{46}$. Knowing speciation in the aqueous bulk phase is important when hydrodynamics comes into play, while speciation at the interface controls the kinetics of extraction ${ }^{1}$.

Second, the existence of a surface-induced phase transition was observed and quantified, occurring at considerably lower concentration than indicated in a phase stability map, consisting in the progressive formation of a crust of several micrometers inside the droplet, until all the extractant present is recruited in the crust. Nearly pure oil and a concentrated third phase appear and coexist not only in the bulk, but in droplets of mm-size that are common in any pulsed column used in industrial practice. This behavior may be responsible for a strong reduction of efficiency of a separation device if inappropriate formulations of the $\operatorname{load}^{4}$ are used. It was 
identified via the effect of the "haze" due to a crust forming "near" but not "at" the phase boundary limit ${ }^{47}$. The phenomenon of nucleation of thin crusts ${ }^{5}$ inside the droplets of emulsions, here evidenced directly for the first time, is also of crucial importance for controlling the processes where ceramic precursors are formed directly in the mixing emulsion ${ }^{8}$.

Moreover, in the design of safety margins in operation of metal extraction plants, at industrial or pilot scale, it is crucial to avoid the two- to three-phase transition, because an unexpected occurrence of a third phase would immobilize a full hydro-metallurgic plant. In fact, spontaneous stable emulsification of water and solvent embedded in a liquid crystalline phase would occur, which is in practice nearly impossible or difficult to demulsify. In absence of any "sign" of third phase onset, the safety requirements render necessary to decrease the load in rare earth content, or to increase the acidity: these precautions increase the imprint of process wastes for a given amount or extracted rare earth. The experimental tool used here proved to promptly detect the "approach" of a dangerous formulation and overload of the feed fluid with ions to be separated. Thus, it can contribute to design processes with increased efficiency, decreasing acid concentration required for operation without any decrease in safety of the liquid-liquid extraction/stripping as a whole.

Finally yet importantly, the confocal Fabry-Perot method is calibration-free even in the case of liquid-liquid interfaces and allows direct measurement of surface waves of the order of one millionth of the diameter of the oil droplet investigated. Although the development of this method is only at its infancy, we were able to follow the formation of interfacial films and their dynamics from thicknesses of $1 \mathrm{~nm}$ to some $100 \mu \mathrm{m}$.

The new possibility to directly access low-amplitude surface fluctuations of emulsified processes will also have an impact on quickly optimizing formulations, in order to avoid the 
presence of any type of thickening of the liquid-liquid interface beyond a monolayer, such as multi-layers and crusts slowing down extraction kinetics. 
FIGURES
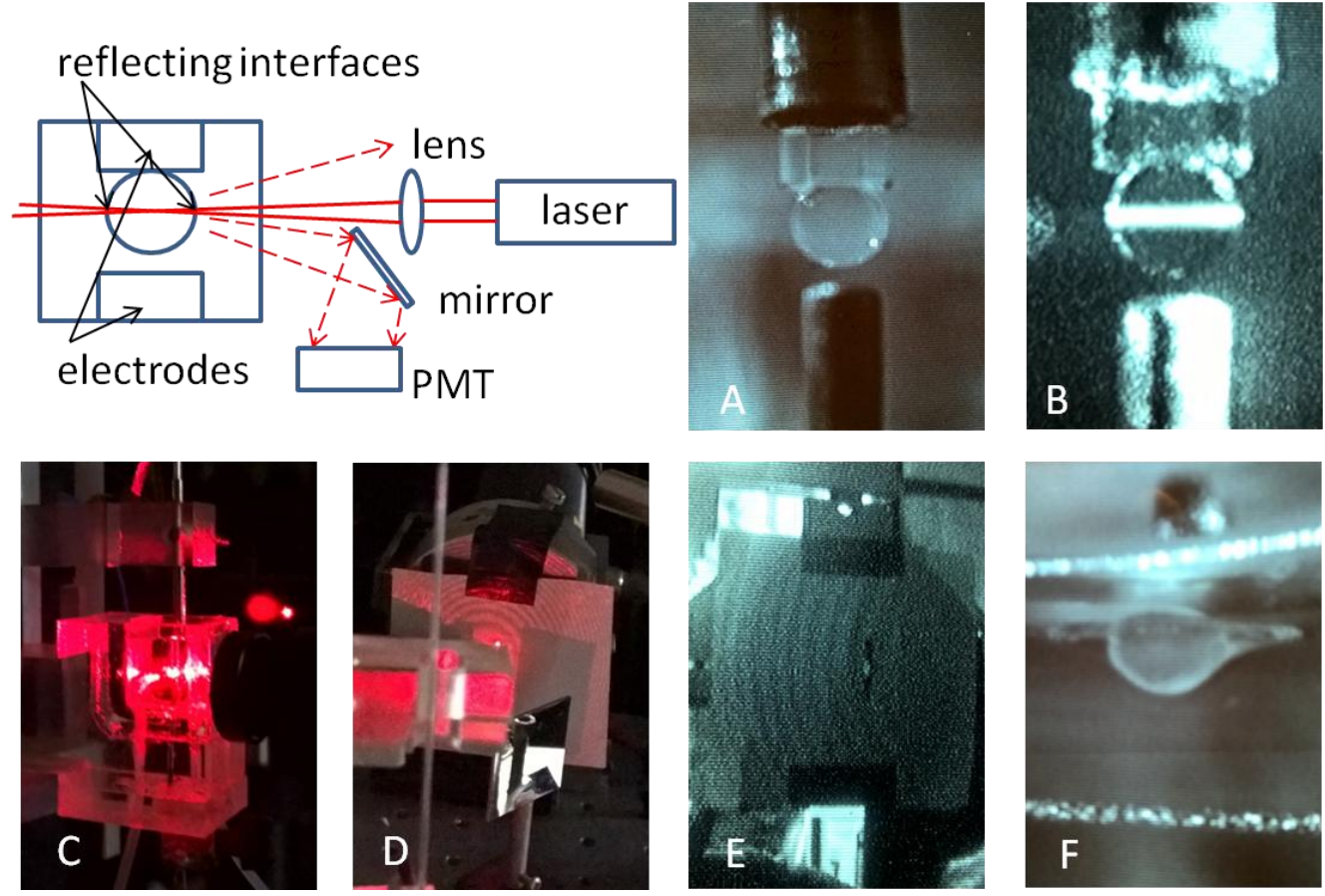

Figure 1. The FPC electro-optic device used to observe pinned oil droplets in water is sketched on the top left. The droplet here studied is located between two electrodes and attached to a glass tube (A), then traversed by a focused laser beam $(80 \mu \mathrm{m})$ in the equatorial direction (B). The laser trace across the TODGA:dodecane drop indicates the presence of reverse aggregates in solution. The drop is hosted in a cell containing the immiscible aqueous fluid at $\mathrm{pH}=3.3(\mathrm{C})$. A mirror (D) sends part of the reflected interference fringes towards the PMT that is placed after a small slit and registers the light falling on the slit (E). Panel (F) shows the solid-like jelly droplet left unbound floating at the cell surface once the third phase smectic lyotropic liquid crystal is formed. 

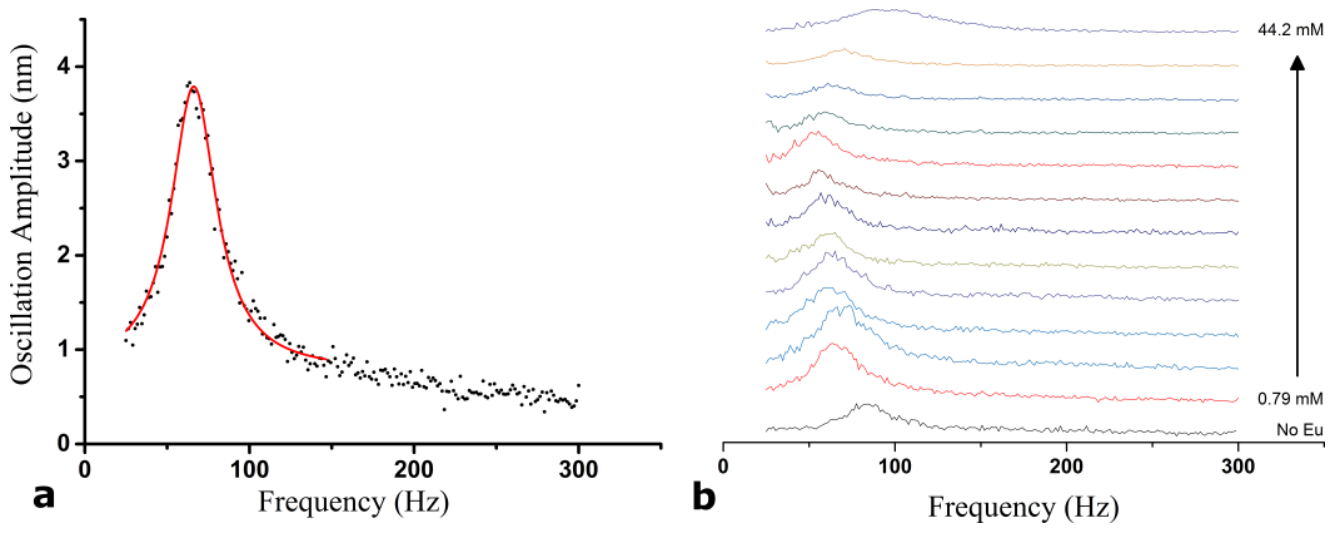

Figure 2. Amplitude spectra. The interferometric nature of the experiment ensures absolute calibration of the measured amplitudes, cf. reference 8. (a) The resonant response of a $0.5 \mathrm{~mm}$ radius drop (30\% TODGA in dodecane) in a $0.79 \mathrm{mM}$ Europium solution at $\mathrm{pH}=3.3$. The amplitude of excitation voltage is 1 Volt and the frequency spans from $30 \mathrm{~Hz}$ to $300 \mathrm{~Hz}$. The full line represents the Lorentz fit to the resonance peak. (b) Sequence of spectra of the $0.5 \mathrm{~mm}$ radius drop (30\% TODGA in dodecane) at increasing Europium salt concentration in the aqueous solvent: (bottom to top) $0,0.79,1.56,2.29,3.0,4.4,7.3,12.1,19.4,26.2,30.0,33.7$, $44.2 \mathrm{mM}$. 


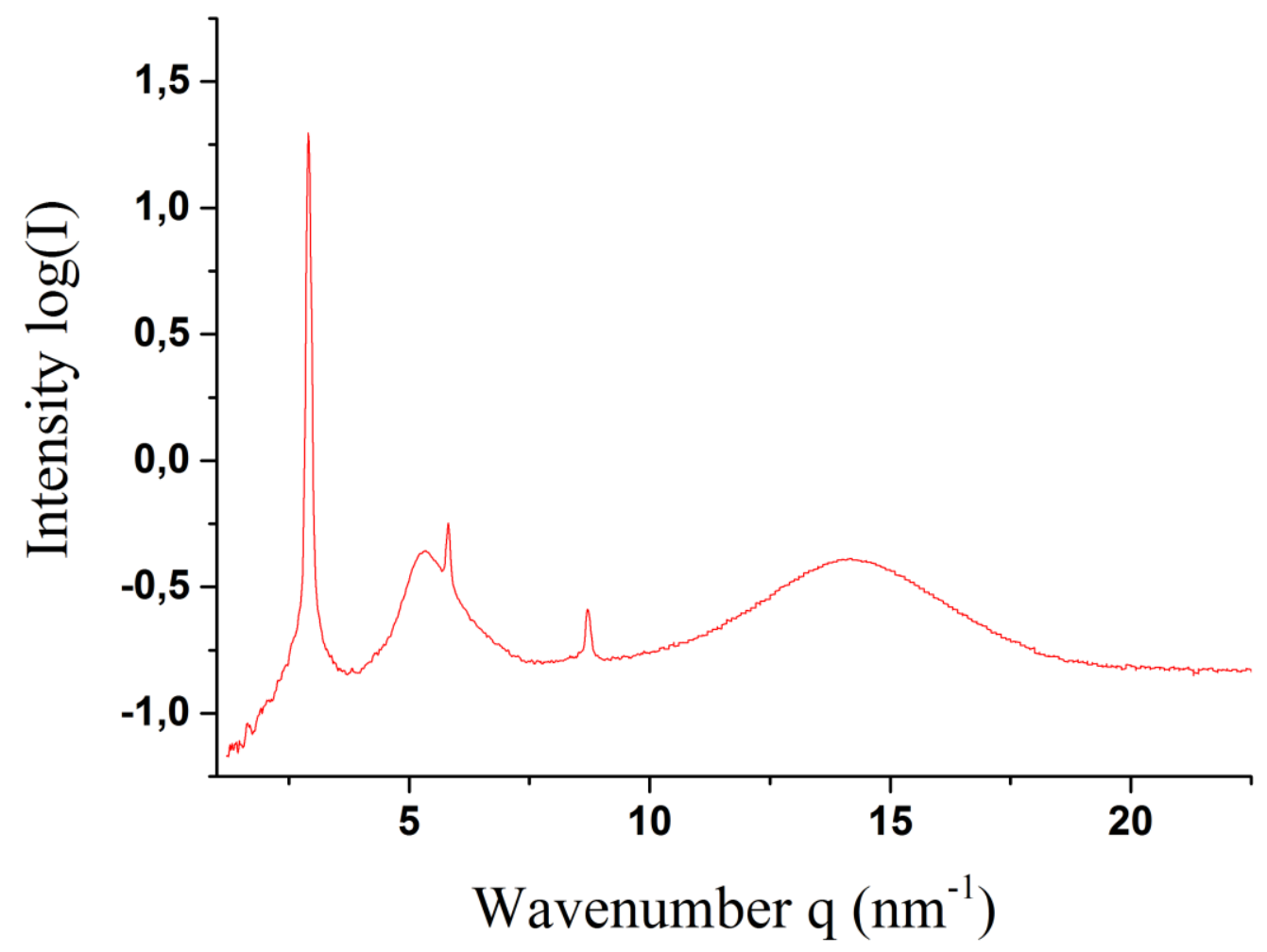

Figure 3. SWAXS intensity of third phase dodecane/TODGA/Europium. The solvent peak is situated around $14 \mathrm{~nm}^{-1}$. The smectic nano-structure yields three extremely sharp pseudo-Bragg peaks with the first order at $2.16 \mathrm{~nm}$ spacing. The broad peak around $5.3 \mathrm{~nm}^{-1}$ is compatible with the stoichiometric TODGA:Eu $=2: 1$ coordination and refers to the average distance of contrasting ions on the residence planes in the smectic structure, while the high-q part of the spectrum with a broad WAXS bump at spacing $4.5 \mathrm{~nm}$ reflects the fluid state of the four hydrophobic chains per extractant. Comparison with the pure dodecane and pure TODGA spectra is provided in the Suppl. Inf. 


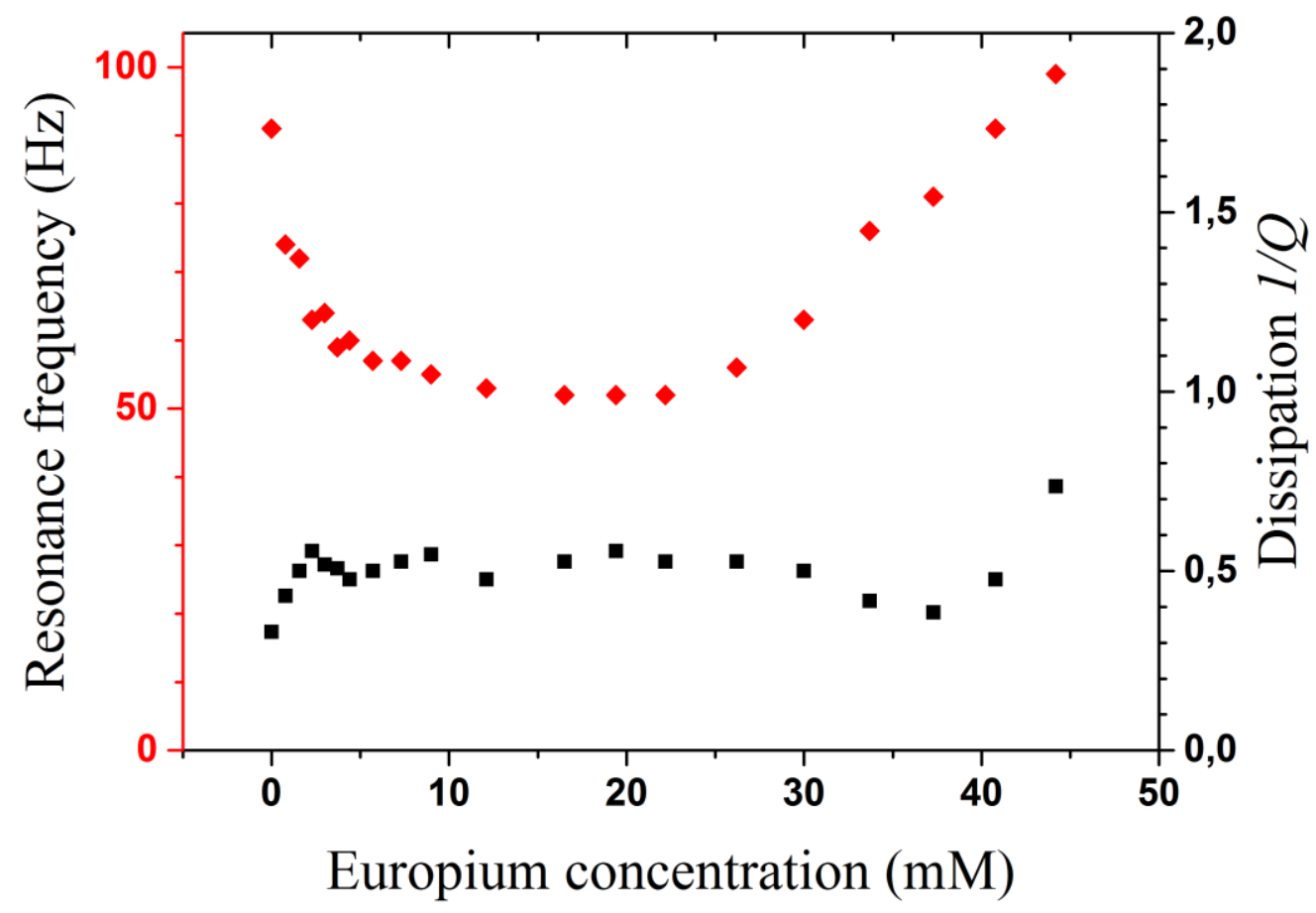

Figure 4. Central frequency of the lowest resonance mode, $l=2$, (red diamonds, left axis) and Eliminato: Resonance central frequency dissipation $1 / Q$ (black squares, right axis) for a $0.5 \mathrm{~mm}$ radius drop (30\% TODGA in dodecane) Formattato: Tipo di carattere: Corsivo versus Europium nitrate salt concentration in the aqueous phase $(\mathrm{pH} 3.3$, adjusted with nitric acid). Dissipation variation is within errors in the whole range. 

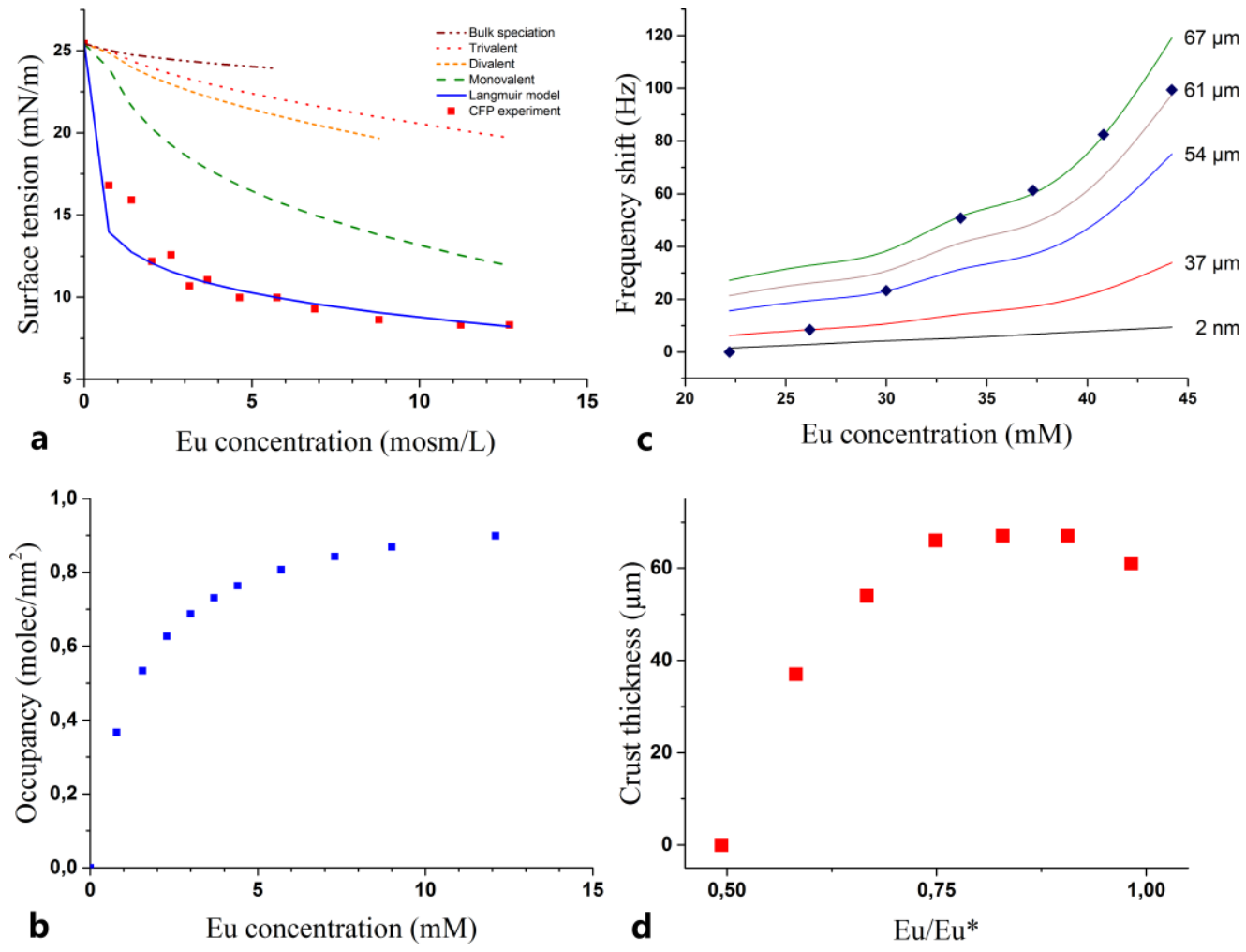

Figure 5. Left side: Surface filling regime. (a) Excess surface tension as derived from frequency shift versus Europium activity measured experimentally (squares), as compared to four possibilities of speciation of Europium ions adsorbed at the interface (see text): bulk speciation with $90 \%$ trivalent Europium and $10 \%$ divalent complexes, trivalent Europium, divalent and monovalent Europium nitrate complexes (Poisson-Boltzmann model), and associated uncharged Europium nitrate quartet complex (Langmuir model). The speciation of Europium adsorbed at the droplet interface is clearly different from that in the bulk. (b) Occupancy of the TODGA surface head-groups (each $1 \mathrm{~nm}^{2}$ ) corresponding to the hypothesis in text. Right side: Crust regime. (c) Increase of droplet resonance frequency at higher Europium concentrations. Experimental data (diamonds) are compared to the crust model adjusting crust thickness. (d) 
Crust thickness evolution as derived from the frequency shift observed and measured values of viscosity and Young modulus as a function of Europium concentration (see Suppl. Inf. Section 2), normalized to the concentration at which third phase transition occurs in a test tube.

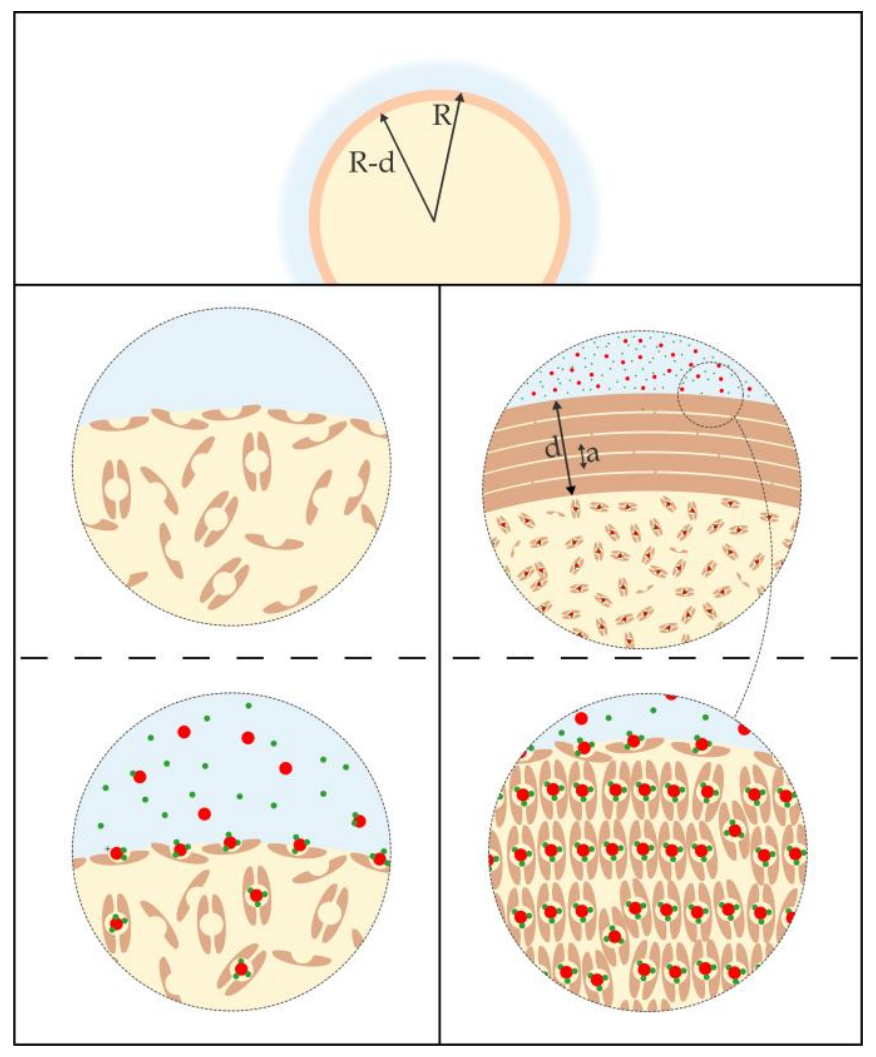

Figure 6. Multi-scale structure of the droplet and nomenclature of parameters. $R$ is the external radius of the droplet; $\mathrm{d}$ the thickness of the interphase, $a$ is the spacing of the smectic structure (when present). The surface area per TODGA molecule at the drop-water interface is $S=1 \mathrm{~nm}^{2}$, as deduced from liquid-liquid surface tension measurements. Left side: Sketch of the drop-water interface in the regime of low concentration of Europium nitrate. For zero Europium nitrate concentration, Debye length is set by background salt and is of the order of the distance between 
extractants. Right side: Sketch in the regime of higher concentration of Europium nitrate, i.e. closer to the third-phase transition, showing the presence of a crust of thickness $d$ with welldeveloped smectic structure with repeat distance $L$ of the order of the molecular length. In the drawing, a 2:1 complexation ratio is used, compatible with SWAXS data of Figure 3: the interconnections between layers act to prevent sliding. 


\section{ASSOCIATED CONTENT}

The following files are available free of charge. Detailed methods found in the supplementary information are about:

1) Calculation of resonance frequencies of a spherical drop with a layered elastic crust.

2) Physico-chemical properties of the Winsor II and third phase of the system TODGA/dodecane/Eu/water.

\section{AUTHOR INFORMATION}

\section{Corresponding Author}

Correspondence should be addressed to JT: johannes.theisen@,cea.fr, +33 (0) 438-786-006.

\section{Author Contributions}

The manuscript was written through contributions of all authors. The theoretical part was written by AR while the confocal Fabry-Perot interferometric experiments were performed by MC, LC and TZ. "Bulk" determinations were performed by MP. Physico-chemical data were calculated by JT. All authors have given approval to the final version of the manuscript.

\section{Funding Sources}

The research leading to these results has received funding from the European Research Council under the European Union's Seventh Framework Programme (FP/2007-2013) / ERC Grant Agreement n. [320915] "REE-CYCLE".

\section{ACKNOWLEDGMENT}


Luc Belloni is acknowledged for the access to specific software solving non-linear implicit equations evaluating surface adsorption of ions including complexation and charge regulation as well as one non-electrostatic term using primitive model of continuous solvent. Julien Rey is acknowledged for the access to data acquired during his $\mathrm{PhD}$. Olivier Diat, Jean-François Dufrêche, Jean-Christophe Gabriel are acknowledged for helpful discussions. The ERC grant "REE-CYCLE" is acknowledged for support of the work presented in materials and travels, as well as for the employment of Johannes Theisen.

\author{
ABBREVIATIONS \\ TODGA: TetraOctylDiGlycolAmide, $\mathrm{C}_{36} \mathrm{H}_{72} \mathrm{~N}_{2} \mathrm{O}_{3}$. \\ CFP: $\quad$ Confocal Fabry-Perot
}




\section{REFERENCES}

(1) Rydberg, J.; Cox, M.; Musikas, C.; Choppin, G. R. Solvent Extraction Principles and Practice; Marcel Dekker: New York (USA), 2004. , Pe Formation of Surface Multilayers at the Air-Water Interface From Sodium Diethylene Glycol Monoalkyl Ether Sulfate/AlC13 Solutions: the Role of the Alkyl Chain Length. Langmuir 2013, 29, 12744-12753.

Xu, H.; Thomas, R. K.; Penfold, J.; Li, P. X.; Ma, K.; Welbourne, R. J. L.; Roberts, D. W.; Petkov, J. T. The Impact of Electrolyte on the Adsorption of the Anionic Surfactant Methyl Ester Sulfonate at the Air-Solution Interface: Surface Multilayer Formation. Journal of Colloid and Interface Science 2018, 512, 231-238.

Arm, S. T.; Jenkins, J. A. Haze Formation and Behavior in Liquid-Liquid Extraction Processes. In Supramolecular Structure in Confined Geometries; ACS Symposium Series; American Chemical Society: Washington, DC, 2009, 933, 103-118.

Scoppola, E.; Watkins, E.; Li Destri, G.; Porcar, L.; Campbell, R. A.; Konovalov, O.; Fragneto, G.; Diat, O. Structure of a Liquid/Liquid Interface During Solvent Extraction Combining X-Ray and Neutron Reflectivity Measurements. Physical Chemistry Chemical Physics 2015, 17, 15093-15097.

Giustiniani, A.; Drenckhan, W.; Poulard, C. Interfacial Tension of Reactive, Liquid Interfaces and Its Consequences. Advances in Colloid and Interface Science 2017, $1-0$.

Bauer, C.; Bauduin, P.; Dufreche, J. F.; Zemb, T.; Diat, O. Liquid/Liquid Metal Extraction: Phase Diagram Topology Resulting From Molecular Interactions Between Extractant, Ion, Oil and Water. Eur. Phys. J. Spec. Top. 2012, 213, 225 241.

Corti, M.; Bonomo, M.; Raudino, A. New Interferometric Technique to Evaluate the Electric Charge of Gas Bubbles in Liquids. Langmuir : the ACS journal of surfaces and colloids 2012, 28, 6060-6066.

Ding, H. S.; Tong, L. G.; Chen, G. H. A Sensitive and Stable Confocal Fabry-Perot Interferometer for Surface Ultrasonic Vibration Detection. Chinese Physics 2001, $10,730-734$.

Testard, F.; Berthon, L.; Zemb, T. Liquid-Liquid Extraction: an Adsorption Isotherm at Divided Interface? Comptes Rendus Chimie 2007, 10, 1034-1041. Zemb, T.; Bauer, C.; Bauduin, P.; Belloni, L.; Déjugnat, C.; Diat, O.; Dubois, V.; Dufrêche, J.-F.; Dourdain, S.; Duvail, M.; et al. Recycling Metals by Controlled Transfer of Ionic Species Between Complex Fluids: en Route to "Ienaics." Colloid \& Polymer Sci 2014, 293, 1-22.

Vernekar, P. V.; Jagdale, Y. D.; Patwardhan, A. W.; Patwardhan, A. V.; Ansari, S. A.; Mohapatra, P. K. Non-Dispersive Solvent Extraction of Neodymium Using N,N,N',N'-Tetraoctyl Diglycolamide (TODGA). Separation Science and Technology 2014, 49, 1541-1554.

Modolo, G.; Asp, H.; Vijgen, H.; Malmbeck, R. Demonstration of a TODGA/TBP Process for Recovery of Trivalent Actinides and Lanthanides From a PUREX Raffinate. Solvent Extraction and Ion Exchange 2008, 26, 62-76. Tachimori, S.; Sasaki, Y.; suzuki, S.-I. Modification of TODGA-N-Dodecane 
Solvent with a Monoamide for High Loading of Lanthanides(III) and Actinides(III). Solvent Extraction and Ion Exchange 2007, 20, 687-699.

Nave, S.; Testard, F.; Coulombeau, H.; Baczko, K.; Larpent, C.; Zemb, T. Ternary Phase Diagrams of a Thermoreversible Chelating Non-Ionic Surfactant. Physical Chemistry Chemical Physics 2009, 11, 2700-2707. Small-Angle Scattering Techniques. J Appl Crystallogr 2003, 36, 373-380. Kolesnichenko, I. V.; Anslyn, E. V. Practical Applications of Supramolecular Chemistry. Chem. Soc. Rev. 2017, 46, 2385-2390.

Cantu', L.; Raudino, A.; Corti, M. An Interferometric Technique to Study Capillary Waves. Advances in Colloid and Interface Science 2017, 247, 23-32.

Obrien, R. W.; Ward, D. N. The Electrophoresis of a Spheroid with a Thin DoubleLayer. Journal of Colloid and Interface Science 1988, 121, 402-413.

Delgado, A. V.; González-Caballero, F.; Hunter, R. J.; Koopal, L. K.; Lyklema, J. Measurement and Interpretation of Electrokinetic Phenomena (IUPAC Technical Report). Pure and Applied Chemistry 2005, 77.

Corti, M.; Pannuzzo, M.; Raudino, A. Trapping of Sodium Dodecyl Sulfate at the Air-Water Interface of Oscillating Bubbles. Langmuir 2015, 31, 6277-6281.

Reed, J.; Ramakrishnan, S.; Schmit, J.; Gimzewski, J. K. Mechanical Interferometry of Nanoscale Motion and Local Mechanical Properties of Living Zebrafish Embryos. ACS Nano 2009, 3, 2090-2094.

Lobo, V. M. M.; Quaresma, J. L. Handbook of Electrolyte Solutions; 1989.

Binnemans, K.; Jones, P. T.; Blanpain, B.; Van Gerven, T.; Yang, Y.; Walton, A.;

Buchert, M. Recycling of Rare Earths: a Critical Review. Journal of Cleaner

Production 2013, 51, 1-22.

Déjugnat, C.; Dourdain, S.; Dubois, V.; Berthon, L.; Pellet-Rostaing, S.; Dufrêche, J.-F.; Zemb, T. Reverse Aggregate Nucleation Induced by Acids in Liquid-Liquid Extraction Processes. Phys Chem Chem Phys 2014, 16, 7339-7349.

Karmakar, A.; Bley, M.; Duvail, M.; Dufreche, J. F.; Zemb, T. Combined Supramolecular and Mesoscale Modelling of Liquid-Liquid Extraction of Rare Earth Salts. Submitted 2017.

Nilsson, M.; Nash, K. L. Review Article: a Review of the Development and Operational Characteristics of the TALSPEAK Process. Solvent Extraction and Ion Exchange 2007, 25, 665-701.

Bauer, C.; Bauduin, P.; Diat, O. Ion Extractant as Cosurfactant at the Water-Oil Interface in Microemulsions. Tenside Surfactants Detergents 2010, 47, 307-311. Bowcott, J. E.; Schulman, J. H. Emulsions Control of Droplet Size and Phase Continuity in Transparent Oil- Water Dispersions Stabilized with Soap and Alcohol. Berichte Der Bunsen-Gesellschaft-Physical Chemistry Chemical Physics 1955, 59, 283-290.

Winsor, P. A. Hydrotropy, Solubilisation and Related Emulsification Processes. Trans. Faraday Soc. 1948, 44, 376-398.

Osseo-Asare, K. Aggregation, Reversed Micelles, and Microemulsions in LiquidLiquid Extraction: the Tri-N-Butyl Phosphatediluent-Water-Electrolyte System. Advances in Colloid and Interface Science 1991, 37, 123-173.

Erlinger, C.; Belloni, L.; Zemb, T.; Madic, C. Attractive Interactions Between 
Reverse Aggregates and Phase Separation in Concentrated Malonamide Extractant Solutions. Langmuir 1999, 15, 2290-2300.

Nave, S.; Modolo, G.; Madic, C.; Testard, F. Aggregation Properties of N,N,N',N'Tetraoctyl-3-Oxapentanediamide (TODGA) in N-Dodecane. Solvent Extraction and Ion Exchange 2007, 22, 527-551.

Dufreche, J. F.; Zemb, T. Effect of Long-Range Interactions on Ion Equilibria in Liquid-Liquid Extraction. Chemical Physics Letters 2015, 622, 45-49.

Ninham, B. W.; Parsegian, V. A. Electrostatic Potential between Surfaces Bearing Ionizable Groups in Ionic Equilibrium with Physiologic Saline Solution. J. Theor. Biol. 1971, 31, 405-428.

Leontidis, E. Chaotropic Salts Interacting with Soft Matter: Beyond the Lyotropic Series. Current Opinion in Colloid \& Interface Science 2016, 23, 100-109. Leontidis, E.; Aroti, A.; Belloni, L. Liquid Expanded Monolayers of Lipids as Model Systems to Understand the Anionic Hofmeister Series: 1. a Tale of Models. $J$ Phys Chem B 2009, 113, 1447-1459.

Charbonnel, M. C.; Flandin, J. L.; Presson, M. T.; Atalante2000, J. M.; 2000.

Thermodynamics Properties of Complexation and Extraction of Ln (III) by Diamides. iaea.org.

Sukenik, S.; Sapir, L.; Harries, D. Balance of Enthalpy and Entropy in Depletion Forces. Current Opinion in Colloid \& Interface Science 2013, 18, 495-501. Vlachy, N.; Jagoda-Cwiklik, B.; Vácha, R.; Touraud, D.; Jungwirth, P.; Kunz, W. Hofmeister Series and Specific Interactions of Charged Headgroups with Aqueous Ions. Advances in Colloid and Interface Science 2009, 146, 42-47.

Yuan, W.; Laprade, E. J.; Henderson, K. J.; Shull, K. R. Formation and Mechanical Characterization of Ionically Crosslinked Membranes at Oil-Water Interfaces. Soft Matter 2014, 10, 1142-1150.

Ferri, A.; Dong, W. F.; Miller, R.; Möhwald, H. Elastic Moduli of Asymmetric Ultrathin Free-Standing Polyelectrolyte Nanocomposites. Macromolecules 2006, 39, 1532-1537.

Rozairo, D. P.; Croll, A. B. Using the Sessile Drop Geometry to Measure Fluid and Elastic Block Copolymer Interfaces. Langmuir 2015, 31, 1303-1311.

Diethert, A.; Koerstgens, V.; Magerl, D.; Ecker, K.; Perlich, J.; Roth, S. V.; MuellerBuschbaum, P. Structure and Macroscopic Tackiness of Ultrathin Pressure Sensitive Adhesive Films. Acs Applied Materials \& Interfaces 2012, 4, 3951-3958.

Schenck, D. M.; Fiegel, J. Tensiometric and Phase Domain Behavior of Lung Surfactant on Mucus-Like Viscoelastic Hydrogels. ACS Applied Materials \& Interfaces 2016, 8, 5917-5928.

Rey, J.; Dourdain, S.; Berthon, L.; Jestin, J.; Pellet-Rostaing, S.; Zemb, T. Synergy in Extraction System Chemistry: Combining Configurational Entropy, Film Bending, and Perturbation of Complexation. Langmuir 2015, 31, 7006-7015. Bu, W.; Yu, H.; Luo, G.; Bera, M. K.; Hou, B.; Schumann, A. W.; Lin, B.; Meron, M.; Kuzmenko, I.; Antonio, M. R.; Soderholm, L.; Schlossman, M. L., Observation of a Rare Earth Ion-Extractant Complex Arrested at the Oil-Water Interface During Solvent Extraction. ACS J Chem Phys B 2014, 118, 10662-10674.

Jehannin, M.; Charton, S.; Corso, B.; Möhwald, H.; Riegler, H.; Zemb, T. Structured Solvent Effects on Precipitation. Colloid \& Polymer Sci 2017, 288, 489-10. 
FOR TABLE OF CONTENTS ONLY

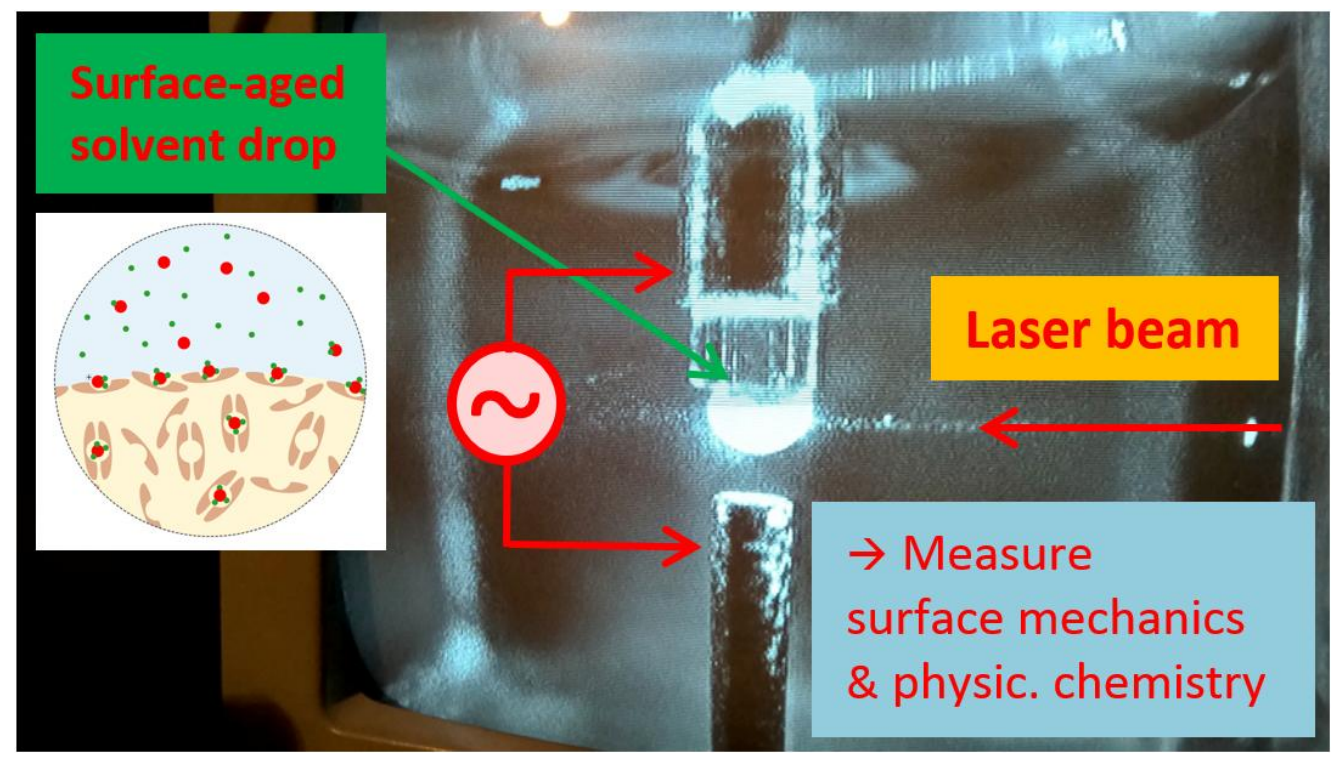

